\title{
Wahrnehmen. Aushalten. Be-zeichnen. Begrenzen.
}

\author{
Zum Umgang mit Angst in der Seelsorge
}

Katja Dubiski

"Ich pack' das nicht mit der Prüfung." (in der Beratung)

"Keine Eltern mehr. Keine Arbeit. Keine Freunde. Wie soll es jetzt nur weitergehen?» (im Trauergespräch)

"Ich habe keine Angst vor dem Tod, aber Angst vor dem Sterben.» (im Krankenhaus)

"Ohne meinen Vater macht nichts mehr Sinn." (im Gespräch mit Jugendlichen)

"Ich will niemandem zur Last fallen." (beim Geburtstagsbesuch)

"Ich habe solche Angst, dass so etwas wieder passiert. Ich kann mich kaum über all das Gute freuen, dass ich gerade erlebe." (im Taufgespräch)

Angst ist Thema in der Seelsorge. In manchen Gesprächen wird sie explizit benannt und liegt als Thema sozusagen auf dem Tisch. In anderen Situationen wird sie nicht benannt, ist aber umso deutlicher zu spüren, wenn Menschen erzählen, was ihnen durch den Kopf geht und was sie tief im Herzen bewegt. Deshalb ist es angemessen und richtig, dass die Frage nach der Angst als "Lebensthema" in poimenischer und pastoralpsychologischer Literatur bearbeitet wird. Es ist eher verwunderlich, dass dies nicht viel häufiger und ausführlicher in der Seelsorge-Literatur erfolgt.

Denn Seelsorge ist Begleitung im Namen Gottes in allen Lebenslagen, also auch in Angst. Ihre Hilfe liegt dabei vor allem im zwischenmenschlichen Beistand, der theologisch als menschlicher Ausdruck der Liebe Gottes gedeutet werden kann. Durch den religiösen Bezug der Seelsorgerin oder des Seelsorgers erhält ein Gespräch die Signatur einer Öffnung zur Transzendenz hin. Für die Seelsorge-Praxis bedeutet der Auftrag der Begleitung in erster Linie, die Lebenslagen bzw. deren Erleben durch das Gegenüber möglichst offen und dadurch im besten Falle auch möglichst genau wahrzunehmen. Nur wenn ich den Standpunkt meines Gegenübers möglichst präzise »orten « kann, kann ich mich begleitend in dessen Nähe stellen und von dort aus den entsprechenden Blickwinkel auf das Leben nachvollziehen. 
Um der Frage nach dem Umgang mit Angst in der Seelsorge nachzugehen, werde ich deshalb nachfolgend in einem ersten Schritt danach fragen, was soziologische, psychologische und theologische Konzepte zu diesem Thema beizutragen haben: An welchen Stellen und in welcher Hinsicht sind deren Erkenntnisse furr Theorie und Praxis der Seelsorge hilfreich? Vor diesem Hintergrund stelle ich dann zweitens Überlegungen zum seelsorgerlichen Umgang mit Angst an.

\section{Wissenschaftliche Wahrnehmungen von "Angst «}

Die Poimenik als Praxistheorie der Seelsorge achtet darauf, Wahrnehmung zu schulen - im interdisziplinären Dialog besonders mit Soziologie und Psychologie, innerdisziplinär durch theologische Reflexion sowie ihres Selbstverständnisses insgesamt. Auch im Hinblick auf Angst geht es darum, den Wahrnehmungshorizont durch eine Breite an entsprechenden Theorien und Deutungsmöglichkeiten zu erweitern und an Praxisbeispielen den eigenen Standpunkt auszuloten, um ihn nachvollziehbar logisch und sachlich korrekt begründen zu können.

\subsection{Soziologie}

Die Soziologie sensibilisiert für die "poimenische Großwetterlage«: Vor welchem gesellschaftlichen Hintergrund treten individuelle Ängste auf? Und inwiefern steht individuelles Angsterleben mit den gesellschaftlichen Rahmenbedingungen in Verbindung?

Zunächst ist mit dem Kultursoziologen Jörn Ahrens festzuhalten, dass es keine ausgearbeitete Soziologie der Angst gibt: "Zwar wird der Topos der >Angst immer wieder bemüht, aber in der Regel eher als scheinbar selbstverständlicher Terminus, der ebenso selbstevident wie assoziationsoffen in Argumentationen verwendet und jedenfalls nirgends mit der nötigen Sorgfalt geklärt wird. «1 In ganz unterschiedlichen Theorien und Ansätzen wird Angst jedoch explizit oder implizit auf unterschiedliche Weise und in durchaus bedeutenden

1 Jörn Ahrens, Angst: Eine (kultur-)soziologische Perspektive, in: Alfred Schäfer/ Christiane Thompson (Hg.), Angst, Paderborn 2018, 37-63, hier 39. Die folgende Darstellung der soziologischen Überlegungen beruht auf diesem Text. 
Zusammenhängen thematisiert. Im Anschluss an Ahrens möchte ich einige kurz nennen.

So findet sich im Hinblick auf Angst die ganz grundsätzliche Annahme, dass Angst kulturbildend wirkt. Daraus ergibt sich für unseren westlich-europäischen Kontext letztlich das Paradox, dass "wir zwar in Gesellschaften der Sicherheit leben, diese sich aber zu weiten Teilen über Gefühle der Angst definieren $\aleph^{2}$. Auf diese Weise ist und bleibt Angst kulturell und gesellschaftlich präsent, obgleich und gerade weil immense Anstrengungen geleistet werden, sie zu bändigen.

So wird selbst in der Risikosoziologie die Rede vom "Risiko" von der "Angst" getrennt. Zwar ist stets die Rede von Risiken, aber kaum von Angst, obwohl die entsprechenden Themen (z.B. die Gentechnologie) immens angstbehaftet sind. Auch Ulrich Beck verknüpft in der "Risikogesellschaft" Risiko und Angst nur am Rande explizit miteinander: Indem der Risikogesellschaft das Ideal bzw. die Utopie der Sicherheit zugrunde liegt, stellt sich als gemeinsamer gesellschaftlicher Antrieb die Angst heraus. So bleibt die gesellschaftliche Utopie »eigentümlich negativ und defensiv « ${ }^{3}$, da Menschen vor allem darauf zielen, Schlimmes zu vermeiden. Angst bildet dabei auch einen Ausgangspunkt von Solidarität: »Noch ist aber völlig unklar, wie die Bindekraft der Angst wirkt. ${ }^{4}$ Es gilt, aufmerksam $\mathrm{zu}$ beobachten, als wie stabil sich diese neue Solidarität auf Basis der Angst erweist und inwiefern sie zu rationalem oder irrationalem Handeln führt.

Auch im Rahmen der Soziologie des Selbst spielt Angst an entscheidender Stelle mit. Indem Selbstverwirklichung die gesellschaftliche Norm geworden ist, wird sie zugleich zur Bedrohung. Denn hinter dem Ideal der Selbstverwirklichung steht zugleich »die Angst des Individuums, zu versagen, krank zu sein und als pathologisiertes Selbst den gesellschaftlichen Normen nicht zu genügen. Der allgemeine Selbstverwirklichungsdiskurs ist demnach zu einem angstbewehrten Projekt gesellschaftlicher Integration geworden. $\mathbb{1}^{5}$

Insgesamt wird an Ahrens' Zusammenstellung deutlich, dass Angst zwar gesellschaftlich geächtet wird, zugleich aber gerade deshalb

\footnotetext{
2 Ebd., 45.

3 Ulrich Beck, Risikogesellschaft. Auf dem Weg in eine andere Moderne, Frankfurt 1986, 65 .

4 Ebd., 66.

5 Ahrens, Angst, 58 im Anschluss an Eva Illouz, Die Errettung der modernen Seele. Therapien, Gefühle und die Kultur der Selbsthilfe, Frankfurt 2009.
} 
zahlreiche soziale Zusammenhänge implizit prägt, wenn nicht sogar bedingt.

Zum Thema der Seelsorge wird Angst dementsprechend gerade da, wo die alltägliche Angstbearbeitung nicht gelingt bzw. in Frage steht. Wo Krankheit, Leid, Sterben und Tod nicht verhindert werden können. Wo Selbstverwirklichung in Frage steht. Wo die Solidarität der Nächsten nicht sicher ist.

Die soziologische Sichtweise verhindert, die Ursachen für die Entstehung von Angst ausschließlich im Individuum und seiner oder ihrer Biographie zu verorten und mahnt, gesellschaftliche und politische Kontexte im Blick zu behalten, wenn von Angst gesprochen wird. ${ }^{6}$

\subsection{Psychologie}

Auch in der Psychologie wird die Thematik der Angst in unterschiedlichen Zusammenhängen bearbeitet. Dies sind im Bereich der Grundlagenforschung zum einen Fragen danach, wann, wie und warum Angst auftritt und welche Folgen sie hat. Erkenntnisse der psychologischen Grundlagenforschung sind für die Seelsorge insofern von Interesse, als das Wissen um die Entstehungsprozesse von Angst sowie um deren Konsequenzen die Basis dessen bildet, wie Seelsorger ${ }^{*}$ innen sich sinnvollerweise zu ihr verhalten. Zum anderen ist dies der Bereich der Anwendungsforschung, in der z.B. die Angst im Sinne von Angststörungen sowie deren Bearbeitung eine Rolle spielen.

Aus Perspektive psychologischer Grundlagenforschung ist zunächst die Unterscheidung der Emotion "Angst« vom Persönlichkeitsmerkmal "Ängstlichkeit« von Bedeutung. ${ }^{7}$ So zeigen Studien, dass Menschen in einer Situation der Angst bei Reaktionszeitexperimenten bedrohliche Wörter unterhalb der Wahrnehmungsschwelle schneller wahrnehmen als Menschen, die gerade keine Angst haben. Andere Studien weisen einen hemmenden Effekt von Angst auf Kreativität und analytisches Denken nach. Insgesamt beeinflusst also "Angst"

\footnotetext{
6 Auf diese Bedeutung der Soziologie für die Seelsorge hat als erste hingewiesen Isolde Karle, Seelsorge in der Moderne. Eine Kritik der psychoanalytisch orientierten Seelsorgelehre, Neukirchen-Vluyn 1996.

$7 \mathrm{Zu}$ "Angst" vgl. Lothar Schmidt-Atzert/Martin Peper/Gerhard Stemmler, Emotionspsychologie. Ein Lehrbuch, Stuttgart ${ }^{2} 2014$, insbes. Kap. 5.4. Zu »Ängstlichkeit» vgl. Gerhard Stemmler/Dirk Hagemann/Manfred Amelang/Frank M. Spinath, Differentielle Psychologie und Persönlichkeitsforschung, Stuttgart ${ }^{8} 2016$, insbes. 411.
} 
in den entsprechenden Situationen Aufmerksamkeit, Denken und Verhalten von Menschen.

Das Persönlichkeitsmerkmal "Ängstlichkeit» ist im Unterschied dazu situationsunspezifisch, d.h. hochängstliche Menschen erleben generell häufiger und intensiver Angst als niedrig-ängstliche Menschen. Wie stark die Angst in einer Situation erlebt wird, ist dabei individuell unterschiedlich und variiert von Situation zu Situation. Für die Seelsorge bedeuten diese Ergebnisse, dass zum einen die konkreten Auswirkungen von Angst auf die Einschätzung von und den Umgang mit der Situation im Blick zu behalten sind. Zum anderen gilt es zu berücksichtigen, dass Menschen Angst unterschiedlich stark und unterschiedlich oft erleben und die Seelsorgerin hier nicht unversehens von sich auf ihr Gegenüber schließen kann.

Die Klinische Psychologie und die Psychotherapieforschung befassen sich im Hinblick auf Angst mit verschiedenen Arten von Angststörungen. Konkret sind dies Phobien, Panikstörung, generalisierte Angststörung, Zwangsstörung, posttraumatische und akute Belastungsstörungen. ${ }^{8}$ In diesem Zusammenhang liefern die unterschiedlichen Ätiologiekonzeptionen der Psychologie verschiedene Deutungen dessen, wie Angststörungen entstehen. Die therapeutischen Modelle der unterschiedlichen psychologischen Paradigmen schließen daran mit verschiedenen Vorgehensweisen an. So fragen psychodynamische Theorien danach, welcher verdrängte Konflikt die eigentliche Ursache der Angst ist und bemühen sich darum, diesen aufzudecken und zu bearbeiten. Lern- bzw. verhaltenstheoretische Theorien hingegen gehen davon aus, dass Angst erlernt wurde und setzen darauf, dass - je nach vorliegender Störung - u.a. durch Konfrontation, Entspannungsübungen oder eine Steigerung der Kompetenzgefühle die Angst wieder abgebaut, d.h. verlernt werden kann.

Die Bandbreite der Konzeptionen macht deutlich, dass es neben der unterschiedlichen Sprache vor allem eine unterschiedliche Akzentuierung der Bedeutung von Angst insgesamt ist, die verschiedene Konsequenzen für die Seelsorge zeitigt. Es macht für den Fortgang eines Gesprächs einen großen Unterschied, ob ein Seelsorger von einer weiten Verbreitung von Angst und einer noch weiter verbreiteten Abwehr von Angst ausgeht - oder ob eine Seelsorgerin, wenn ihr Gegenüber »Angst« explizit zum Thema macht, rein funktional und kleinschrittig nachfragt, wozu diese Angst führt und was deshalb möglicherweise ihre Funktion ist.

\footnotetext{
8 Hierzu und zum folgenden vgl. Gerald C. Davison/John M. Neale/Martin Hautzinger, Klinische Psychologie. Ein Lehrbuch, Weinheim ${ }^{7} 2007,148-199$.
} 
Exkurs: „Angst» in psychoanalytisch orientierten Seelsorge-Konzeptionen

Im Anschluss an die Psychoanalyse hat die Poimenik in der Vergangenheit eine ausgeprägte Aufmerksamkeit dafür entwickelt, dass der Umgang mit Ängsten von verschiedenen Abwehrmechanismen geprägt ist. ${ }^{9}$ So akzentuiert z.B. Klaus Winkler in seinen Überlegungen zur Angst als Thema der Seelsorge besonders die Position Sigmund Freuds:Verdrängung führt zu Angst - und umgekehrt führt Angst zurVerdrängung. ${ }^{10}$ Michael Klessmann betont, dass Menschen durch Verleugnung und Verdrängung möglicherweise keine Worte und darüber hinaus auch kein klares Empfinden mehr für Angst zur Verfügung haben. ${ }^{11}$ Und auch Jürgen Ziemer geht von ähnlichen Annahmen aus: "Angst ruht auf dem Grund fast jeden seelsorglichen Problems [...] Man muss immer mit ihr rechnen. Aber mit der Angst fällt in der Regel niemand ins Haus. Und die direkte Frage nach der Angst löst oft Abwehr, Hilflosigkeit oder Achselzucken aus, manchmal auch Ärger. ${ }^{12}$

Es ist offensichtlich, dass Annahmen dieser Art das konkrete seelsorgerliche Vorgehen prägen, da beinahe überall mit verdrängter Angst gerechnet wird - auch da, wo sie nicht explizit vom Gegenüber benannt wird. Die Annahme einer quasi-ubiquitären Existenz verdrängter Angst kann allerdings dazu führen, die expliziten wörtlichen Aussagen des Gegenübers als nur sekundär bzw. als Ausdruck von Abwehr erscheinen zu lassen.

In den eingangs genannten Beispielen wird die Angst in den Selbstäußerungen thematisiert. Damit steht sie als Überschrift über jedem einzelnen der dann erfolgten Gespräche. In diesen Fällen ist eine generelle Annahme von Verdrängung nicht notwendig, um angemessen mit der geäußerten Angst umgehen zu können.

\subsection{Theologie}

Anders als in der Vergangenheit werden heute Angstlosigkeit und Glaube nicht mehr einfach gleichgesetzt. ${ }^{13}$

\footnotetext{
9 Vgl. Dietrich Stollberg, Vom Umgang mit der Angst in der Seelsorge, in: Pastoraltheologie 80 (1991), 352-362, hier 358f.

${ }^{10}$ Vgl. Klaus Winkler, Seelsorge, Berlin ${ }^{2} 2000,306-308$.

${ }^{11}$ Vgl. Michael Klessmann, Seelsorge. Begleitung, Begegnung, Lebensdeutung im Horizont des christlichen Glaubens. Ein Lehrbuch, Neukirchen-Vluyn ${ }^{4} 2012,227$.

12 Jürgen Ziemer, Seelsorgelehre, Göttingen ${ }^{4} 2015,274$.

13 Vgl. dazu Ulrich H. J. Körtner, „Um Trost war mir sehr bange«. Angst und Glaube, Krankheit und Tod, in: Angst. Theologische Zugänge zu einem ambivalenten Thema,
} 
Vielmehr bildet die Annahme von Gottes Mit-Sein mit den Menschen die theologische Grundlage für den Umgang mit Angst. Gunda Schneider-Flume knüpft in diesem Zusammenhang an die Erinnerung der Geschichte Gottes an, durch die eine neue Zeitperspektive eingeführt und die Geschichte Gottes "als Erfahrung gegen die Erfahrung, als Wirklichkeit gegen die Wirklichkeit geltend gemacht ${ }^{14}$ wird. Der Blick zurück in Dankbarkeit ermutigt zum Blick nach vorn in Hoffnung. So kann christlicher Glaube "zu Angstfähigkeit ermutigen, zu der Fähigkeit, Gefahren wahrzunehmen und vernünftig damit umzugehen, getragen von demVertrauen, daß keine Angst gottverlassen und deshalb ohne Hoffnung ist. ${ }^{15}$

Dieser Aspekt des Mit-Seins Gottes wird ergänzt durch die Hoffnung auf die "endgültige Überwindung ${ }^{16}{ }^{16}$ der Angst, z.B. bei Kirsten Huxel. Deutlich wird auch hier, dass Glaube nicht mit Angstfreiheit gleichzusetzen ist.

So ist jedoch noch undeutlich, wie in der Seelsorge mit der ganz aktuell Lebensmöglichkeiten einschränkenden - Angst von Menschen umzugehen ist. Denn der mit der Hoffnung verbundene mögliche Trost schon in der Gegenwart kann sich zwar einstellen - es besteht jedoch aus seelsorgerlicher Perspektive das Risiko, dass ein zu früher Verweis auf die Hoffnung die Angst des Gegenübers letztlich übergeht. Für die Seelsorge hilfreich ist in dieser Hinsicht die Argumentation Ulrich Körtners, der für den Umgang mit Angst im Glauben die Begriffe Hoffnung und Mut voneinander abgrenzt: Auf Basis der Unterscheidung von Angst und Furcht betont Körtner den Mut als Gegenstück zur Angst: »Die Angst ist also die Bedingung dafür, daß Furcht und Hoffnung entstehen können. Beide haben ihren Grund in der Angst. Als Affekte der Erwartung gehen Furcht und Hoffnung nicht nur auf Objekte zu, sondern füllen auch den leeren Raum der Zukunft mit endlichen Erwartungen und Wunschbildern. ${ }^{17}$ Daraus folgt: "Nicht Hoffnung, sondern Mut ist die Überwindung der Angst. ${ }^{18}$ Glaube ist also "Mut zum fraglichen Sein [...], der ganz im Heute lebt, ohne der Gegenwart $\mathrm{zu}$ verfallen, und über den Tag hinaus zu hoffen wagt, und wär es

\footnotetext{
hg. v. Ulrich H. J. Körtner, Neukirchen-Vluyn 2001, 69-86, hier 73-79.

${ }^{14}$ Gunda Schneider-Flume, Angst und Glaube, in: ZThK 88 (1991), 478-495, hier 493 [Hervorhebungen im Original].

${ }^{15}$ Ebd., 494.

${ }^{16}$ Kirsten Huxel, Das Phänomen Angst. Eine Studie zur theologischen Anthropologie, in: NZSTh 47 (2005), 35-57, hier 56.

${ }^{17}$ Ulrich H. J. Körtner, Weltangst und Weltende. Eine theologische Interpretation der Apokalyptik, Göttingen 1988, 136.

${ }^{18}$ Ebd., 137.
} 
auch der letzte « ${ }^{19}$. Mut und Hoffnung stehen damit für zwei Zeitorientierungen: Mut ist die Orientierung auf das Jetzt und Hoffnung die Orientierung auf das, was kommt - mit Auswirkungen auf das Jetzt. Körtner selbst akzentuiert den Mut, lenkt damit den Blick auf die Gegenwart und buchstabiert aus, wie der Angst zu begegnen ist: zunächst im Aushalten, im »in der Angst sein« - getragen von einer Hoffnung, die den Blick zugleich über sie hinaus weitet.

Für die Seelsorge bedeutet dies: Eine vorschnelle Rede von Hoffnung führt dazu, das Jetzt und die akute Angst nicht ernst zu nehmen - und damit letztlich das Gegenüber und sein Erleben abzuwerten. Seelsorger*innen benötigen vielmehr Wissen über Formen und Mechanismen von Angst und eine gewisse Selbstreflexion. Darüber hinaus ist eine theologische Orientierung darüber notwendig, inwiefern Angst zum menschlichen Leben dazugehört oder auch, inwiefern sie unnötiges Leid verursacht und deshalb zu bekämpfen ist.

\section{Angst in der Seelsorge - Dimensionen einer Haltung}

Die vorangegangenen Analysen haben deutlich gemacht, wie vielschichtig die Herangehensweisen an Angst und die Erklärungsmodelle zu ihrer Entstehung und zum Umgang mit ihr sind. Was bedeutet all dies nun für die Praxis in der Seelsorge? Wie kann eine Seelsorgerin ihrem geängstigten Gegenüber begegnen? Wie kann ein Seelsorger im Bewusstsein um seine eigene Angst seinem Gegenüber hilfreich beiseite stehen? Wie können Seelsorger*innen sich gemeinsam mit Hilfesuchenden in aller Solidarität der Angst stellen?

Ich möchte Angst als Thema der Seelsorge nachfolgend mit den vier Aspekten der Überschrift »Wahrnehmen. Aushalten. Be-zeichnen. Begrenzen." bearbeiten. In der konkreten Situation können sich diese Aspekte ebenso in wenigen Worten realisieren wie in einem langen Gesprächskontakt. Ihre Reihenfolge ist bewusst gewählt, jedoch sind sie nicht als Abfolge abgeschlossener Phasen vorzustellen.Vielmehr bilden sie Dimensionen, die im Verlauf des Gesprächs bestehen bleiben und einander ergänzen. Zusammengenommen bilden sie die Grundlage einer seelsorgerlichen Haltung zur Angst.

${ }^{19}$ Körtner, "Um Trost war mir sehr bange«, 86. 


\subsection{Wahrnehmen}

Wahrnehmung erfolgt nicht objektiv, sondern ist weitreichend von Vorwissen und Vorannahmen geprägt. Je breiter das Vorwissen und je reflektierter diese Vorannahmen, desto deutlicher wird, was in der Wahrnehmung eigentlich geschieht. Dieser Reflexion dienen die oben ausgeführten Modelle und Überlegungen der Soziologie, der Psychologie sowie der Systematischen Theologie. Und auch die folgenden Überlegungen spielen sich in erster Linie unabhängig vom konkreten Seelsorgekontakt ab, sei es in der organisierten Seelsorgeaus- oder -weiterbildung oder in der individuellen Reflexion des eigenen seelsorgerlichen Handelns.

Dabei führt dieser erste Schritt der Wissensaneignung über Entstehung von und Umgang mit Angst im Idealfall zur Reflexion eigener impliziter Vorannahmen auf Seiten der Seelsorger*innen - also der Reflexion dessen, welchen Modellen von gesellschaftlichen und psychischen Vorgängen eine Seelsorgerin folgt oder wie ein Seelsorger sich den Zusammenhang bestimmter menschlicher Aktionen und Reaktionen vorstellt: Gehe ich grundsätzlich von einem großen Anteil verdrängter Angst in Psyche und Gesellschaft aus? Spielt gar die Todesangst die zentrale Rolle überhaupt, wenn es um die Erklärung der Entstehung von Religiosität geht? Lohnt es sich, im Angesicht von Angst in der Seelsorge deren Inhalt nachzugehen oder ihrer Funktion? Was geschieht durch die geschilderte Angst - was wird möglich und was wird verhindert? Sollte sich dies ändern? Wie wäre dies zu ändern? Die Antworten von Seelsorger*innen auf diese Fragen nehmen Einfluss darauf, wie sie den Verlauf eines Gesprächs bzw. Gesprächskontaktes gestalten.

Die Auseinandersetzung mit der poimenischen Literatur fuihrt zu der geschärften Wahrnehmung, dass die frühen Modelle therapeutischer und beratender Seelsorge im Schwung der Seelsorgebewegung tendenziell zu weit gingen im Hinblick auf eine quasitherapeutische Deutung und Bearbeitung von Angst im Rahmen der Seelsorge. Diese Gefahr gilt es, auch heute im Blick zu behalten. Denn wenn sich die Angst aus einem laienpsychologischen Blick als pathologisch im Sinne einer Angststörung erweist, ist die Empfehlung einer professionellen psychotherapeutischen Bearbeitung der zentrale Schritt, ohne das Gespräch mit der Person abzulehnen oder abzubrechen. In dieser Hinsicht gilt es, das Bewusstsein um die eigenen Grenzen zu pflegen und nicht selbst therapeutisch zu agieren.

In den oben genannten Beispielen ist Seelsorge jedoch dazu herausgefordert, die genannte existenzielle Angst genauso zu nehmen, wie sie sich zeigt. Dem entsprechen alle oben genannten soziologi- 
schen und psychologischen Modelle zur Entstehung von Angst, die je auf ihre Weise und in ihrer Sprache den Blick dafür schärfen, dass Angst sinnvoll ist: »Angst darf sein. $\|^{20}$

Freilich bildet diese kognitive Herangehensweise nur die eine Seite dessen, was Wahrnehmung bzw. Schulung in Aus- und Weiterbildung im Bereich der Seelsorge ausmacht. Denn nicht nur das eigene Verständnis von Angst, sondern auch die persönlichen Ängste der Seelsorger*innen selbst beeinflussen die Wahrnehmung von und den Umgang mit der Angst des Gegenübers. Die Seelsorgebewegung hatte bereits in den 1960er Jahren darauf aufmerksam gemacht. Die Seelsorgeausbildung der Kirchen betont - besonders in Form der Klinischen Seelsorgeausbildung KSA - bis heute besonders, dass auch und gerade Selbstwahrnehmung und -erfahrung entscheidende Faktoren sind, wenn es um die Wahrnehmung des Gegenübers geht, und dass diese zu einem großen Teil in der Praxis und anhand praktischer Erfahrungen zu erarbeiten sind. Hier hat die Arbeit mit Gesprächsprotokollen ihren Ort und leistet die kirchliche SeelsorgeAusbildung in der zweiten Ausbildungsphase sowie in Fort- und Weiterbildungen seit langem einen entscheidenden Beitrag.

Mit diesen hauptsächlich in der Ausbildung ablaufenden Prozessen der Wissensaneignung und Reflexion sowie der Selbsterfahrung sind bereits zentrale Schritte dafür getan, in der Seelsorgesituation selbst die Angst und die Ängste des Gegenübers wahrzunehmen.

Dabei liegt der Blick auf verschiedenen Fragen: Inwiefern nehme ich Angst bei meinem Gegenüber wahr? Neben dieser Frage nach impliziter Angst stehen Fragen, die an die explizite Nennung von "Angst" anschließen: Welche Angst wird genannt? Woher kommt sie und wozu führt sie? Erscheint mir die Angst angemessen oder unangemessen? Wie komme ich zu dieser Deutung? Was ist in diesem Zusammenhang mein Auftrag als Seelsorgerin?

\subsection{Aushalten}

Ernst nehmen. Geltenlassen. Zuhören. Annehmen. Tolerieren. Diese und andere Begriffe fallen als Bezeichnung eines zweiten Aspektes in poimenischen Texten zu Angst als Thema der Seelsorge. ${ }^{21}$ Hier soll der Umgang mit Angst als "aushalten" im Sinne von "tolerieren", also hinnehmen und dulden, pointiert werden. Anders als bei der Bezeichnung "annehmen" ist damit jede positive Konnotation vermieden. Denn manche der in den obigen Beispielen genannten

${ }^{20}$ Stollberg,Vom Umgang mit der Angst in der Seelsorge, 360.

${ }^{21}$ Vgl. z.B. Klessmann, Seelsorge, 229f. und Ziemer, Seelsorgelehre, 275. 
Ängste sind auch für die Seelsorgerin schwer zu ertragen. Eine nichtpathologische existenzielle Angst des Gegenübers hat ihre Gründe und macht auch den Seelsorger in gewisser Weise sprachlos. Eine Seelsorge, die sich selbst in erster Linie als Begleitung versteht, wird darüber nicht hinweggehen. Angst gehört zum menschlichen Leben dazu und ist als solche wahr- und ernst zu nehmen:»Angst zu haben, bedeutet immer, in seinen Entfaltungsmöglichkeiten beschränkt zu sein, und ist mit Leid verbunden. $\|^{22}$

Die Ermutigung zur Angstfähigkeit bedeutet für die Seelsorge also nicht, Angst zu verniedlichen oder weich zu zeichnen. Es geht nicht darum, unmittelbar einen Ausweg zu suchen oder gar bereits zu kennen. Es geht vielmehr zunächst um den Weg in der Angst. Dietrich Stollberg beschrieb als Aufgabe der Seelsorge das "nonverbale Dabeisein im Sinne einer solidarischen Begleitung auf dem Wege der Angst « ${ }^{23}$.Jürgen Ziemer baut diesen Gedanken später noch stärker psychologisch aus und betont das schlichte Dasein im Sinne einer »Erfahrung von menschlicher Nähe und Solidarität ${ }^{24}$.

Mit diesen Aspekten des "Wahrnehmens" und "Aushaltens" hat Seelsorge in manchen Situationen möglicherweise schon getan, was sie tun kann. In anderen Situationen kann sich jedoch der Aspekt des zunächst tendenziell "sprachfreien" Aushaltens von Angst auch mit Sprache verbinden.

\subsection{Be-zeichnen}

Indem Angst als "Angst" benannt wird, erhält sie einen Namen und gewinnt an Kontur und Struktur. Durch diese "Diagnose« wird sie umrissen, umrandet, quasi be-zeichnet. ${ }^{25}$ Es tut Menschen gut, ihre deutlich gefühlte Angst im Gespräch zu benennen, sie in den Raum zu stellen und sie zum Thema zu machen. Dabei ist das Benennen der Angst selbst bereits als Folge dessen zu sehen, was sich in der Seelsorge durch Wahrnehmen und Aushalten ereignen kann. Eine auf diese Weise realisierte seelsorgerliche Haltung der Akzeptanz, Empathie und Echtheit schafft eine angstfreie Atmosphäre. ${ }^{26}$ In

\footnotetext{
${ }^{22}$ Irmtraud Fischer, Angst und Glaube, in: Angst. Medizin, Psychologie, Gesellschaft, hg. v. Peter Zwanzger, Berlin 2019, 27-31, hier 27.

${ }^{23}$ Stollberg,Vom Umgang mit der Angst in der Seelsorge, 361.

${ }^{24}$ Ziemer, Seelsorgelehre, 277 [im Original tw. hervorgehoben].

${ }^{25}$ Zur Bedeutung des Erzählens verwendet auch Ziemer den Begriff "Bezeichnung" (ebd., 275) und beschreibt als Voraussetzung dessen eine Atmosphäre des Vertrauens (ebd., 274).

${ }^{26}$ Zum Zusammenhang von Gesprächshaltung und angstfreier Atmosphäre vgl. Helga Lemke/Wilhelm Thürnau, Personzentrierte Psychotherapie und Seelsorge, in:
} 
dieser kann sich das Gegenüber ohne Angst vor Verurteilung frei äußern. Menschen zeigen sich verletzlich, weil sie spüren, dass sie es dürfen, ohne etwas zu verlieren. Mit ihren ehrlichen und offenen Äußerungen stellen sie ihr Angsterleben in den Raum und machen so möglich, dass nun etwas damit geschieht.

In erster Linie wird dies »das Gespräch über die Angst und die Ängste ${ }^{27}$ sein. Schon bei Luther findet sich dieser Umgang mit Angst und diese Art der Angstbewältigung: Erzählen hilft. ${ }^{28}$ So wird die Angst Wort für Wort und Gedanke für Gedanke genauer bezeichnet. Dabei akzentuiert die Dimension der "Be-zeichnung" den Blick auf die Gegenwart in ihrem Gewordensein, weniger auf den Blick in die Vergangenheit um ihrer selbst willen.

Darüber hinaus sind es spezifisch religiöse Be-zeichnungen, die den Umgang mit Angst als Thema der Seelsorge bestimmen können. Hier bietet der Rückgriff auf die Psalmen eine Möglichkeit, Menschen eine sehr bildhafte, emotional ausgeprägte und doch zugleich fremde Sprache für ihre eigene Angst anzubieten. ${ }^{29}$ Irmtraud Fischer stellt besonders die Klagepsalmen mit ihrer "Schilderung der Not vor Gott $\aleph^{30}$ als Umgang mit Angst dar. Indem Menschen darin vor Gott bringen, was sie bewegt, zeichnet sich ein erster Schritt aus der Angst ab. Fischer weist aber darüber hinaus darauf hin, dass dies in den Psalmen nicht in Form spontaner Gebete erfolgt, sondern die Psalmen wohlüberlegte und institutionell verankerte Gebetsformen des Jerusalemer Tempels darstellen. In diesen erfolgt in den allermeisten Fällen ein Umschwung von der Klage zum Dank - und auch wieder zurück. Dargestellt wird also ein Prozess, der »in kreisender Annäherung die Betenden strategisch zu einer Bewältigung « ${ }^{31}$ führt, indem sie sich daran erinnern, was Menschen vor ihnen mit Gott erlebt haben. Dieser komplexen Struktur der Klagepsalmen schreibt Fischer therapeutische Qualität zu. Sie machen deutlich, dass der Umgang mit Angst nicht als einfaches und einmaliges Geschehen zu beschreiben, sondern realistisch als lebenslange und wiederkehrende Aufgabe wahrzunehmen ist.

Auch im Neuen Testament wird Angst immer wieder als Realität auch des christlichen Lebens benannt und zugleich in den Horizont der Hoffnung gestellt. Besonders ist dies in Joh 16,33 der

\footnotetext{
Handbuch der Seelsorge, hg. v. Wilfried Engemann, Leipzig ${ }^{3} 2016$, 330-346, hier $335 f$.

${ }^{27}$ Stollberg,Vom Umgang mit Angst in der Seelsorge, 361.

${ }^{28}$ Vgl. Michael Klessmann, Angst und Angstbewältigung als Gegenstand praktischtheologischer Reflexion, Waltrop 1998, 14 f.

${ }^{29}$ Vgl. ebd., 35.

${ }^{30}$ Fischer, Angst und Glaube, 28.

${ }^{31}$ Ebd., 29.
} 
Fall: »In der Welt habt ihr Angst, aber seid getrost, ich habe die Welt überwunden." (Joh 16,33). Aber auch bei Paulus kommt dies zum Ausdruck: »Wer will uns scheiden von der Liebe Christi? Trübsal oder Angst oder Verfolgung oder Hunger oder Blöße oder Gefahr oder Schwert? (Röm 8,35). Es geht in beiden Bibelworten darum, »der Angst standzuhalten. Glaube ist nicht Angstlosigkeit, sondern die Fähigkeit mit ihr zu leben «².

Vielleicht können diese alten Worte heute gerade durch ihre Fremdheit eine seelsorgerliche Ressource sein, die es im Umgang mit der Angst sensibel und kreativ zu nützen gilt.

\subsection{Begrenzen}

Das Be-zeichnen der Angst führt dazu, sie fassbar zu machen und schon dadurch, dem Grauen, das sie in sich trägt, aktiv zu begegnen. Darüber hinaus kann mit dem „Begrenzen“ der Angst noch eine weitere, eher quantitativ konnotierte Dimension in der Seelsorge hinzutreten. Diese soll nun abschließend zunächst im Hinblick auf spezifisch religiöse Ressourcen, dann mit Fokus auf die seelsorgerliche Gesprächsführung generell dargestellt werden.

Aus theologischer Perspektive ist Angst Teil der Schöpfung und damit "relativ und begrenzt "33 $^{3}$. Sie ist nicht das Letzte und sie hat nicht das letzte Wort. Dies schwingt in allen oben genannten theologischen Überlegungen und biblischen Texten bereits mit. Die Begrenzung der Angst erfolgt z.B., indem Angst in Form der Klage vor Gott gebracht wird. So steht sie als innerweltliches, menschliches Phänomen vor Gott. Dies gilt über die Klage hinaus für das Gebet insgesamt. ${ }^{34}$ Im Gebet - nicht nur in der Klage - kommt die Angst vor Gott, dem Schöpfer und der Mutter allen Lebens, zur Sprache und wird bereits durch diese Konstellation selbst relativiert. In der Seelsorge gilt es, mit diesen Möglichkeiten des Gebets bzw. des Verweises auf biblische Texte sensibel umzugehen. Ähnliches gilt für den großen Fundus an Liedern, die sich mit der Angst befassen. Im Evangelischen Gesangbuch trägt ein ganzer Abschnitt im Stammteil die Überschrift "Angst und Vertrauen" (EG 361-383). Zahlreiche weitere und jüngere Lieder zum Thema finden sich in den Regional-

\footnotetext{
32 Ziemer, Seelsorgelehre, 273. Ziemer nennt diese beiden und noch zahlreiche andere Bibelstellen.

${ }^{33}$ Stollberg,Vom Umgang mit Angst in der Seelsorge, 362.

${ }^{34}$ Zum Gebet als ,"Trostform« vgl. Ziemer, Seelsorgelehre, 277f. Vgl. auch Klessmann, Seelsorge, 230 .
} 
teilen, z.B. »Fürchte dich nicht«, »Meine engen Grenzen«, »Du Gott stützt mich«, »Ich lobe meinen Gott«, »Nada te turbe«.

Jenseits dieser spezifisch religiösen Formen kann es daneben in der Seelsorge auch geboten sein, die Angst selbst so weit wie möglich zu begrenzen. Um die Möglichkeiten einer solchen Begrenzung auszuloten, ist es hilfreich, der Rede von der Angst mit einer präzisen Wortwahl den Akzent der Veränderung zu verleihen. ${ }^{35}$ Dies könnte z.B. heißen, auf die Aussage "X macht mir Angst« mit der Rückfrage "Und was machen Sie mit der Angst?" zu reagieren oder die Aussage umformuliert und in veränderungsoffener Sprache aufzunehmen: "Was fürchten Sie?«. In anderen Kontexten (z.B. der Angst, zur Last zu fallen), könnte man fragen: "Was würden Sie ohne diese Angst denken, fühlen, tun? « So wird die Existenz oder Berechtigung der Angst nicht geleugnet. Auch geht man so nicht davon aus, die Angst würde durch eine bestimmte sprachliche bzw. therapeutische Intervention einfach verschwinden. Vielmehr ist es so möglich, z.B. im Anschluss an Konzeptionen Kognitiver Verhaltenstherapie oder Beratung, den Akzent des Vorgehens auf ein ernsthaftes "Umdenken", auf eine kognitive Umorientierung zu legen. Hierzu können sowohl die konkrete Angst selbst als auch ihre Ursachen etwas ausführlicher in den Blick kommen, bevor dann nach einer entsprechenden Lösung gesucht wird. Oder man geht im Anschluss an systemtherapeutisch oder systemtheoretisch basierte Konzeptionen deutlich spielerischer mit der Angst um - und nimmt ihr vielleicht gerade so einen Teil ihrer Bedrohlichkeit: In der Annahme, dass jede Intervention das bestehende System auf eine vorher nicht absehbare Weise verändert, können hier Seelsorger*innen die Suche nach Ressourcen und Lösungsmöglichkeiten direkt initiieren und das Gespräch auf diese Weise positiv und bestärkend gestalten.

\footnotetext{
${ }_{35}$ Ausführlich dargestellt sind diese Überlegungen in Katja Dubiski, Seelsorge und Kognitive Verhaltenstherapie. Plädoyer für eine psychologisch informierte Seelsorge, Leipzig 2017, 272f. Hinzuweisen ist in diesem Kontext auf Rolf Winiarski, KVT in Beratung und Kurztherapie, Weinheim/Basel ${ }^{2} 2012$; Timm H. Lohse, Das Trainingsbuch zum Kurzgespräch. Ein Werkbuch für die seelsorgliche Praxis, Göttingen 2006; Steve de Shazer/Yvonne Dolan, Mehr als ein Wunder. Lösungsfokussierte Kurztherapie heute, Heidelberg ${ }^{4} 2015$.
} 


\section{Fazit}

Abschließend sei die Thematik in den größeren Kontext eingebettet. Denn der Umgang mit Angst in der Seelsorge endet nicht mit dem einzelnen Seelsorgegespräch.Von daher ist mit Körtner die Aufgabe des Glaubens - und der Seelsorge - als eine zweifache zu beschreiben: Sie besteht "nicht nur im Mut zur Angst, wo sie unaufhebbar ist, sondern ebenso in der Aufklärung über lebensfeindliche Angstmechanismen, in der Vertreibung falscher Ängste, in der Bekämpfung jedes Geschäftes mit der Angst. ${ }^{36}$ Denn gerade die in einem Seelsorgekontakt konkret benannte Angst fordert dazu heraus, auch dagegen anzugehen, dass Angst politisch oder ökonomisch verwendet und so die konkret verängstigten Menschen mit ihrer Angst für andere Zwecke missbraucht werden.

Insgesamt sind für die Frage nach der Angst Überlegungen zum Zusammenhang von Angst und Macht gewinnbringend. Denn Angst macht »buchstäblich ohn-mächtig « ${ }^{37}$. Demgegenüber zielt z.B. Psychotherapie darauf, »die absolute Macht der Angst(symptomatik) in bestimmten Situationen zu beenden und der Angst die Macht zu geben, die ihr eigentlich gebührt, nämlich uns vor bestimmten Situationen im Vorhinein zu warnen. $^{38}$ Seelsorgerlich gewendet heißt dies: Die Relativierung der Angst im Angesicht Gottes gibt Menschen die Macht, sich vom Absolutheitsanspruch der Angst immer wieder zu lösen, um neu befreit ins Leben zu gehen.

- Dr. theol. Katja Dubiski, Dipl. Psych., ist Wissenschaftliche Mitarbeiterin am Lehrstuhl für Praktische Theologie der Ruhr-Universität Bochum. Zu ihren Arbeitsgebieten gehören der Dialog von Praktischer Theologie und Psychologie, insbesondere in den Bereichen Seelsorge und Gender, außerdem Fragen der Anthropologie und der Spiritualität.

${ }^{36}$ Körtner, »Um Trost war mir sehr bange«, 78.

${ }^{37}$ Markos Maragkos, Angst und Macht, in: Angst. Medizin. Psychologie. Gesellschaft, hg. v. Peter Zwanzger, Berlin 2018, 44-53, hier 46 [Hervorhebung im Original].

${ }^{38}$ Ebd., 46f. 